\title{
Steroid-refractory extensive enteritis complicated by ulcerative colitis successfully treated with adalimumab
}

\author{
Shinji Okabayashi ${ }^{1}$, Taku Kobayashi ${ }^{1}$, Tomohisa Sujino ${ }^{1}$, Ryo Ozaki ${ }^{2}$, Satoko Umeda ${ }^{2}$, Takahiko Toyonaga ${ }^{2}$, \\ Eiko Saito ${ }^{1}$, Masaru Nakano ${ }^{2}$, Maria Carla Tablante ${ }^{1}$, Shojiroh Morinaga ${ }^{3}$, Toshifumi Hibi ${ }^{1}$ \\ ${ }^{I}$ Center for Advanced IBD Research and Treatment, Departments of ${ }^{2}$ Gastroenterology and Hepatology and ${ }^{3}$ Diagnostic Pathology, \\ Kitasato University Kitasato Institute Hospital, Tokyo, Japan
}

Extracolonic involvement of the gastrointestinal tract is extremely uncommon in ulcerative colitis (UC) and rarely found in the upper gastrointestinal tract or in postoperative cases since it typically responds to steroids. Here we report a case of UC complicated by extensive ileal inflammation that was refractory to steroids. A 20-year-old man was diagnosed with UC of typical pancolitis without ileal involvement and started treatment with $\mathrm{pH}$-dependent mesalazine and oral prednisolone. Although his symptoms transiently resolved, the condition flared when the steroid dose was tapered down. Computed tomography revealed marked thickening of the ileal wall, and capsule endoscopy and balloon-assisted enteroscopy found diffuse mucosal inflammation with ulcers in the ileum. On the contrary, the inflammation in the colon and rectum was improving. Since the response to the second steroid course was inadequate, treatment with adalimumab and 6-mercaptopurine was initiated and finally achieved clinical and endoscopic remission. The investigation of small intestinal lesions is necessary in patients with UC whose clinical deterioration cannot be explained by colonic lesions. (Intest Res 2017;15:535-539)

Key Words: Ulcerative colitis; Enteritis; Inflammatory bowel disease; Capsule endoscopy; Balloon-assisted enteroscopy

\section{INTRODUCTION}

Ulcerative colitis (UC) is a chronic IBD characterized by diffuse mucosal inflammation involving the colon and rectum and a poorly understood etiology. UC predominantly involves the rectum and spreads proximally, generally sparing the other parts of the digestive tract proximal to the ileocecal valve. However, gastroduodenal lesions ${ }^{1-11}$ and enteritis were also recently reported in cases of UC, including backwash ileitis, ${ }^{12}$ postoperative enteritis, and pouchitis. Moreover, due to technological advances and the development of new de-

Received November 30, 2016. Revised January 11, 2017. Accepted January 16, 2017. Published online March 7, 2017

Correspondence to Taku Kobayashi, Center for Advanced IBD Research and Treatment, Kitasato University Kitasato Institute Hospital, 5-9-1 Shirokane,

Minato-ku, Tokyo 108-8642, Japan. Tel: +81-3-3444-6161, Fax: +81-3-

3444-2530, E-mail: kobataku@insti.kitasato-u.ac.jp

Financial support: None. Conflict of interest: Taku Kobayashi and

Toshifumi Hibi received lecture fees from AbbVie, Kyorin Pharmaceutical Co.,

Ltd, Eisai Co., Ltd, and ZERIA Pharmaceutical Co., Ltd. vices visualizing the entire small intestine, rare cases of small intestinal lesions complicated by UC have recently been reported. ${ }^{13}$

We experienced a rare case of extensive enteritis complicated by unoperated UC in which the extent of involvement was identified by capsule endoscopy and balloon-assisted enteroscopy (BAE). Extensive enteritis complicated by UC is rare, and steroid-refractory enteritis in which remission was achieved with adalimumab was not reported previously.

\section{CASE REPORT}

A 20-year-old man was apparently well until he presented at a local hospital with a 1-month history of frequent bloody diarrhea, intermittent fever, and lower abdominal pain. His symptoms had progressively worsened, thus prompting the consultation (Fig. 1). Colonoscopy revealed diffuse friable mucosa throughout the entire colon and rectum with erosions in the sigmoid colon and rectum but without ileal in-

\footnotetext{
๑ Copyright 2017. Korean Association for the Study of Intestinal Diseases. All rights reserved.

This is an Open Access article distributed under the terms of the Creative Commons Attribution Non-Commercial License (http://creativecommons.org/licenses/by-nc/4.0)

which permits unrestricted non-commercial use, distribution, and reproduction in any medium, provided the original work is properly cited.
} 


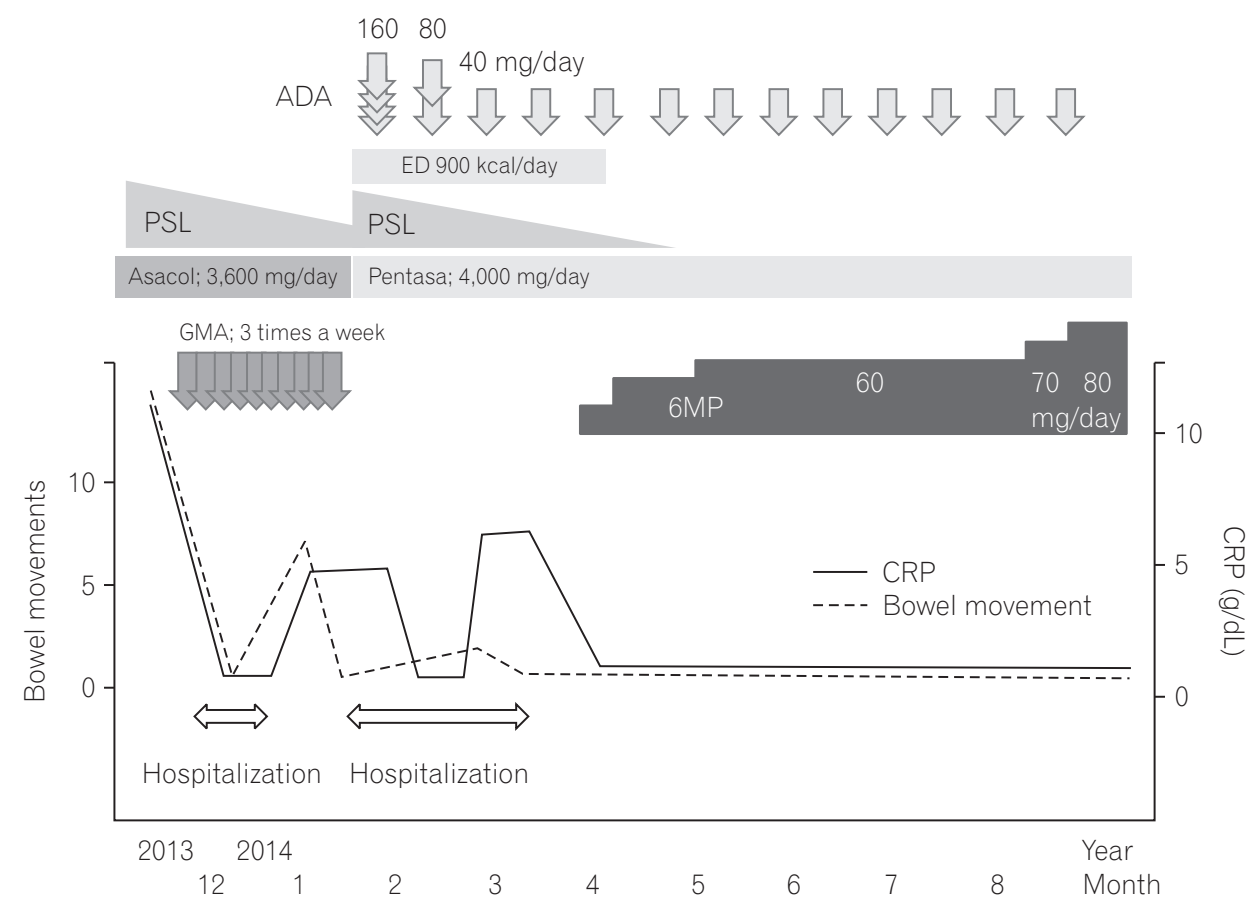

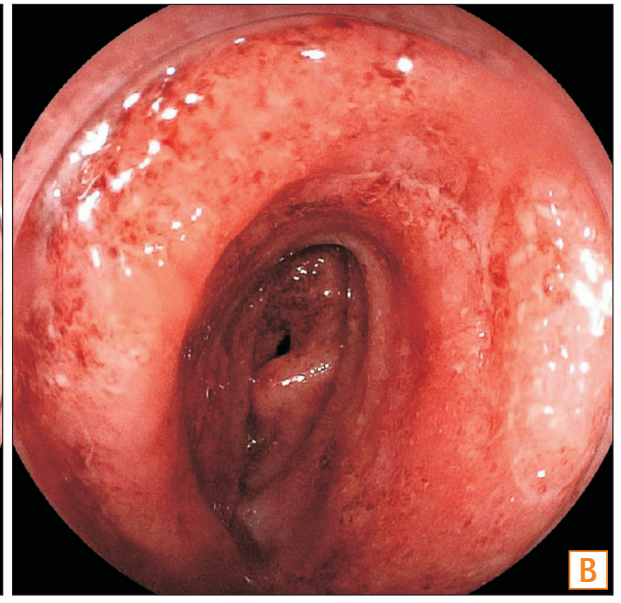

Fig. 1. Clinical course of a patient who achieved clinical remission. ADA, adalimumab; ED, elemental diet; PSL, prednisolone; GMA, granulocyte monocyte apheresis; 6MP, 6-mercatopurine.
Fig. 2. Endoscopic findings prior to diagnosis and treatments of UC. Colonoscopy revealed no inflammation within the terminal ileum (A), and inflammation with diffused friable mucosa throughout the entire colon and rectum with erosions in the sigmoid colon and rectum (B). volvement (Fig. 2), and no significant findings were observed in the small intestine on CT. Histological examinations of the biopsies obtained from the colon revealed moderate chronic inflammation with neutrophilic and lymphoplasmacytic infiltration between intercellular substances, disturbed epithelial cells, decreased goblet cells, and a crypt abscess without granulomas. He was diagnosed with UC and started treatment with mesalazine (Asacol ${ }^{\mathbb{}}$; ZERIA Pharmaceutical Co., Ltd., Tokyo, Japan) 3,600 mg/day. However, his condition deteriorated and he was referred to Kitasato University Kitasato Institute Hospital 2 months after first experiencing symptoms. The patient was a nonsmoker with no significant past medical history or family history of IBD.
On admission, the patient had moderate to severe symptoms, including $>10$ bowel movements per day with bloody diarrhea, lower abdominal pain, and a low-grade fever. Laboratory examinations revealed: white blood cell count, $11,690 / \mu \mathrm{L}$; hemoglobin, $14.4 \mathrm{~g} / \mathrm{dL}$; albumin, $4.6 \mathrm{~g} / \mathrm{dL}$; and CRP, $10.09 \mathrm{~g} / \mathrm{dL}$. Intravenous prednisolone $60 \mathrm{mg} /$ day was administered and tapered by $10 \mathrm{mg}$ per week. He also underwent leukocytapheresis three times a week up to 10 times. His bowel movements decreased to only three times per day with no bloody stools and no associated abdominal pain. He was discharged after 3 weeks of treatment.

The patient continued outpatient care and the steroid was eventually tapered down to $20 \mathrm{mg}$ /day; however, his 

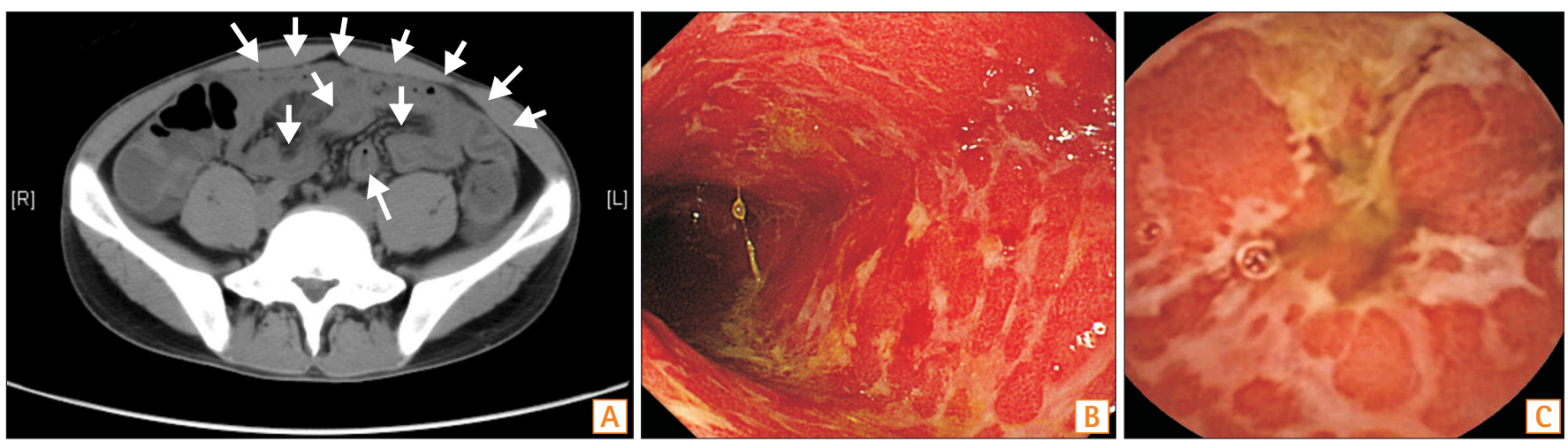

Fig. 3. CT and endoscopic findings of UC after tapering of the steroid treatment. Widespread inflammation in the small intestine was observed via CT ( $A$, arrows). Balloon-assisted enteroscopy revealed severe extensive inflammation with multiple ulcers spreading in the ileum and mild inflammation in the terminal ileum, colon, and rectum (B). Capsule endoscopy discovered diffuse mucosal inflammation with erosions and ulcers in the middle to distal ileum (C).

symptoms relapsed and he was readmitted to the hospital 2 weeks after discharge. At this time, he was febrile $\left(38^{\circ} \mathrm{C}\right)$ and experienced nonbloody diarrhea more than 10 times per day accompanied by mild abdominal pain. Laboratory data on readmission revealed: white blood cell count 16,720/ $\mu \mathrm{L}$ and an increase of CRP from 0.06 to $5.44 \mathrm{~g} / \mathrm{dL}$. We also observed widespread inflammation in the small intestine via CT. Colonoscopy revealed improved mucosal inflammation in the colon and rectum with additional mild diffused inflammation in the terminal ileum compared to the colonoscopic findings before steroid administration; hence, the small intestinal lesion was first suspected to be infectious enteritis, which is usually self-limiting, and the patient was treated with intravenous fluids only. However, no diseasecausing bacteria or viruses were detected by stool culture, and the continued worsening of his symptoms prompted further examinations of the small intestinal lesion identified previously with CT. Capsule endoscopy and BAE confirmed widespread diffuse mucosal inflammation exceeding 100 $\mathrm{cm}$ with multiple ulcers in the ileum with mild inflammation limited to the terminal ileum (Fig. 3) consistent with the CT findings. Biopsies from the ileum and the colon both revealed moderate chronic inflammation with neutrophilic and lymphoplasmacytic infiltration resembling UC (Fig. 4); no granuloma nor characteristic findings of cytomegalovirus infection were discovered.

The patient was diagnosed with extensive enteritis complicated by UC and treated with intravenous mesalazine (Pentasa $^{\circledR}$; Kyorin Pharmaceutical Co., Ltd, Tokyo, Japan) 60 $\mathrm{mg} /$ day instead of Asacol ${ }^{\circledR}$ and an elemental diet was started. However, these treatments proved ineffective and his symptoms lasted more than a month, so he was eventually started

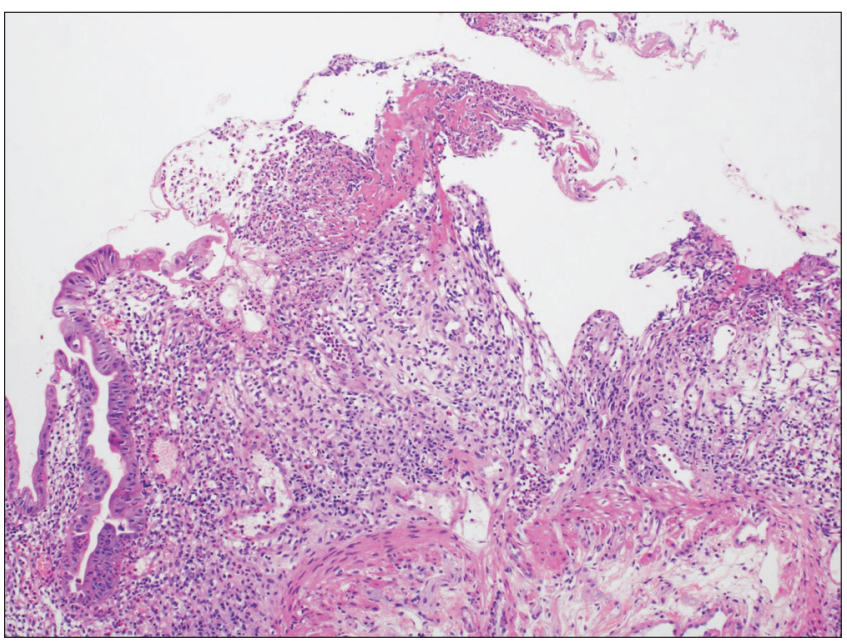

Fig. 4. Biopsy specimen taken from the ileum revealing moderate chronic inflammation with neutrophilic and lymphoplasmacytic inflammation with erosion $\left(\mathrm{HEE}_{1} \times 40\right)$.

on anti-tumor necrosis factor- $\alpha$ treatment. Adalimumab was chosen according to the patient's preference. His symptoms resolved after induction therapy with adalimumab and he was again discharged from the hospital.

Two months after tapering of the dosage from $60 \mathrm{mg} /$ day, the steroid treatment was successfully discontinued and his condition was maintained on adalimumab, 6-mercatopurine, and mesalazine. He has been in remission for 2 years both clinically and endoscopically confirmed by capsule endoscopy, BAE (Fig. 5), and colonoscopy.

\section{DISCUSSION}

Here we presented an extremely rare case of UC with ex- 


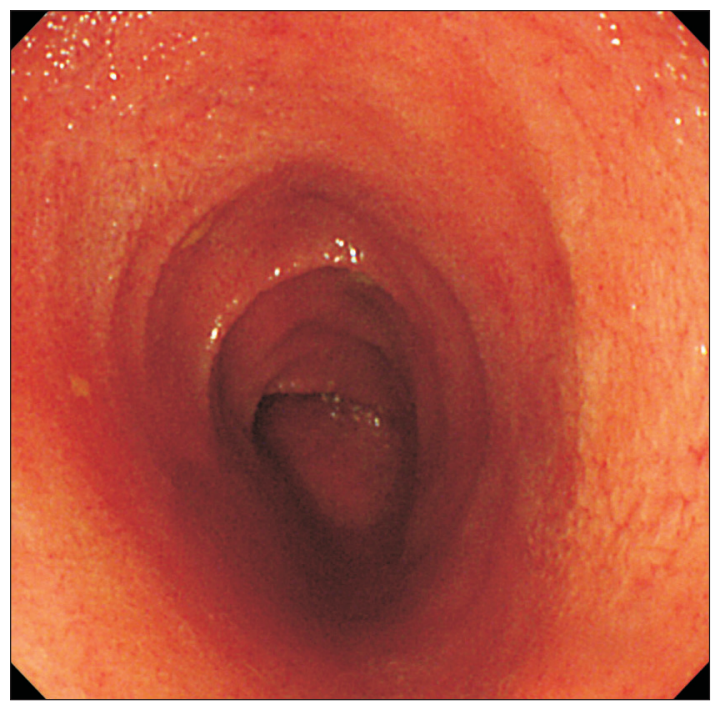

Fig. 5. Endoscopic findings after treatment for extensive involvement in the small intestine. Balloon-assisted enteroscopy revealed remission in the small intestine.

tensive inflammation in the small intestine in which the inflammation of the colon and rectum was under control. The entire digestive tract was examined from the mouth to the anus using various endoscopic techniques including esophagogastroduodenoscopy, colonoscopy, BAE, and capsule endoscopy. The extent of involvement in the digestive tract was successfully determined, especially the extent and severity of the inflammation in the small intestine. The patient successfully achieved remission both clinically and endoscopically with adalimumab, 6-mercatopurine, and mesalazine.

Cases of UC complicated by extensive involvement in the stomach, duodenum, and small intestine are rare, and 29 cases were previously reported in the literature (Table 1$) .^{1-11,14-18} \mathrm{Al}-$ most all of the cases had duodenitis, which was not seen in our case. A total of 16 cases were confirmed to have jejunitis or ileitis, all were postoperative enteritis or pouchitis, and only 7 cases had no history of colectomy; however, our case did not have these characteristic complications. Outcomes for extensive involvement of the stomach, duodenum, and small intestine are reported to be generally successful with a good response to steroid treatment; however, such treatment was ineffective in our case and the patient was successfully treated with adalimumab, 6-mercatopurine, and mesalazine. In addition, in contrast to backwash ileitis, which commonly causes a limited extent of inflammation in the terminal ileum due to a malfunction of the ileocecal valve when the inflammation in the cecum is severe, ${ }^{12}$ our case demonstrated severe inflammation exceeding $100 \mathrm{~cm}$ in the ileum with mild inflammation limited to the terminal ileum and inflam-
Table 1. Clinical Characteristics of 29 Patients with UC Complicated by Extensive Involvement in the Stomach, Duodenum, and Small Intestine in Previous Studies

\begin{tabular}{lc}
\hline \multicolumn{1}{c}{ Characteristic } & $\begin{array}{c}\text { Value } \\
(\mathbf{n = 2 9 )}\end{array}$ \\
\hline Age (yr) & $30(3-61)$ \\
Male sex & 69.2 \\
Duration of UC (yr) & $4(0-46)$ \\
History of colectomy & $22(75.9)$ \\
Extent of UC & \\
Pancolitis & $21(72.4)$ \\
Left-sided colitis & $5(17.2)$ \\
Proctitis & 0 \\
Unknown & $3(10.3)$
\end{tabular}

Extensive involvement in stomach, duodenum, and small intestine

$\begin{array}{lr}\text { Gastritis } & 10(34.5) \\ \text { Duodenitis } & 27(93.1) \\ \text { Jejunitis } & 7(24.1) \\ \text { lleitis } & 13(44.8)\end{array}$

Treatment for extensive involvement in stomach, duodenum and small intestine

$\begin{array}{lc}\text { Steroid } & 25(86.2) \\ \text { Mesalazine or/and salazosulfapyridine } & 8(27.6) \\ \text { Immunomodulator (azathioprine or 6-mercatopurine) } & 10(34.5) \\ \text { Cyclosporine } & 1(3.4) \\ \text { Anti-TNF- } \alpha \text { (infliximab or adalimumab) } & 2(6.9) \\ \text { Outcome of extensive involvement in the stomach, } & \\ \text { duodenum, and small intestine } & 19(65.5) \\ \text { No relapse } & 6(20.7) \\ \text { Relapse } & 2(6.9) \\ \text { Refractory or died } & 2(6.9) \\ \text { Unknown }\end{array}$

Values are presented as median (range), percent, or number (\%). TNF- $\alpha$, tumor necrosis factor- $\alpha$.

mation of the cecum that had improved after the previous treatments.

The possibility of CD in this case was excluded because macroscopic and histological findings did not satisfy any of the Japanese or international diagnostic criteria. ${ }^{19} \mathrm{We}$ suspect that the gut microbiota plays an important role in inflammation in postoperative enteritis or pouchitis since antibiotics and probiotics are effective against them. Even without surgery, we suspected that the small intestinal inflammation might be associated with gut bacterial flora. 
Gut bacteria are more abundant in the distal ileum than the jejunum and proximal ileum, ${ }^{20}$ and in the present case, the extent of inflammation relatively corresponded with the distal ileum, where bacteria is more abundant. In addition, a study done by Higurashi et al. ${ }^{13}$ showed that small intestinal lesions tend to be observed on capsule endoscopy in the distal ileum in patients with UC, a finding that is consistent with that in our case. However, in the present case, the enteritis improved without antibiotics or probiotics, and the patient achieved remission after only immunoregulation.

The prevalence of small intestinal lesions in UC may be underestimated because the small intestine is not routinely examined; technological advances and the development of new devices such as capsule endoscopy and BAE would enable physicians to more precisely identify the small intestinal lesions complicated by UC. In contrast, it is known that performing endoscopy during active disease can cause disease worsening in some patients. In our case, we considered the BAE safe because improvement of the colonic lesions was confirmed by colonoscopy prior to the BAE. A decision about the need for further investigations of the small intestinal lesions by BAE may require careful consideration; however, it is especially useful in cases of clinical deterioration that cannot be explained by colorectal lesions.

\section{REFERENCES}

1. Sasaki M, Okada K, Koyama S, et al. Ulcerative colitis complicated by gastroduodenal lesions. J Gastroenterol 1996;31:585589.

2. Ikeuchi H, Hori K, Nishigami T, et al. Diffuse gastroduodenitis and pouchitis associated with ulcerative colitis. World J Gastroenterol 2006;12:5913-5915.

3. Endo K, Kuroha M, Shiga H, et al. Two cases of diffuse duodenitis associated with ulcerative colitis. Case Rep Gastrointest Med 2012;2012:396521.

4. Valdez R, Appelman HD, Bronner MP, Greenson JK. Diffuse duodenitis associated with ulcerative colitis. Am J Surg Pathol 2000;24:1407-1413.

5. Chiba M, Ono I, Wakamatsu H, Wada I, Suzuki K. Diffuse gastroduodenitis associated with ulcerative colitis: treatment by infliximab. Dig Endosc 2013;25:622-625.

6. Terashima S, Hoshino Y, Kanzaki N, Kogure M, Gotoh M. Ulcerative duodenitis accompanying ulcerative colitis. J Clin Gastroenterol 2001;32:172-175.
7. Shimura T, Inukai M, Yoshioka N, et al. A case of diffuse gastritis and duodenitis associated with ulcerative colitis. Nihon Shokakibyo Gakkai Zasshi 2006;103:30-36.

8. Kawai K, Watanabe T, Nakayama H, Roberts-Thomson I, Nagawa H. Gastrointestinal: small bowel inflammation and ulcerative colitis. J Gastroenterol Hepatol 2005;20:1791.

9. Nakajima M, Nakashima H, Kiyohara K, et al. Case with diffuse duodenitis and enteritis following total colectomy for ulcerative colitis. Nihon Shokakibyo Gakkai Zasshi 2008;105:382-390.

10. Annese V, Caruso N, Bisceglia M, et al. Fatal ulcerative panenteritis following colectomy in a patient with ulcerative colitis. Dig Dis Sci 1999;44:1189-1195.

11. Mitomi H, Atari E, Uesugi H, et al. Distinctive diffuse duodenitis associated with ulcerative colitis. Dig Dis Sci 1997;42:684-693.

12. Goldstein N, Dulai M. Contemporary morphologic definition of backwash ileitis in ulcerative colitis and features that distinguish it from Crohn disease. Am J Clin Pathol 2006;126:365376.

13. Higurashi T, Endo H, Yoneda M, et al. Capsule-endoscopic findings of ulcerative colitis patients. Digestion 2011;84:306-314.

14. Rubenstein J, Sherif A, Appelman H, Chey WD. Ulcerative colitis associated enteritis: is ulcerative colitis always confined to the colon? J Clin Gastroenterol 2004;38:46-51.

15. Corporaal S, Karrenbeld A, van der Linde K, Voskuil JH, Kleibeuker JH, Dijkstra G. Diffuse enteritis after colectomy for ulcerative colitis: two case reports and review of the literature. Eur J Gastroenterol Hepatol 2009;21:710-715.

16. Gooding IR, Springall R, Talbot IC, Silk DB. Idiopathic smallintestinal inflammation after colectomy for ulcerative colitis. Clin Gastroenterol Hepatol 2008;6:707-709.

17. Ectors N, Rutgeerts P, Geboes K, Penninckx F, Desmet V. Ulcerative jejunitis in patients with established ulcerative colitis. Gastroenterology 1994;106:A676.

18. Hoentjen F, Hanauer SB, Hart J, Rubin DT. Long-term treatment of patients with a history of ulcerative colitis who develop gastritis and pan-enteritis after colectomy. J Clin Gastroenterol 2013;47:52-57.

19. Van Assche G, Dignass A, Panes J, et al. The second European evidence-based consensus on the diagnosis and management of Crohn's disease: definitions and diagnosis. J Crohns Colitis 2010;4:7-27.

20. Hao WL, Lee YK. Microflora of the gastrointestinal tract: a review. Methods Mol Biol 2004;268:491-502. 